A 78-year-old man was admitted to hospital due to massive, bright red hematemesis. As he was hemodynamically unstable, vigorous resuscitation procedures were initiated. Esophagogastroduodenoscopy (EGD) showed a large MalloryWeiss lesion and a necrotic polypoid lesion about $4 \mathrm{~cm}$ below the upper esophageal sphincter (Figure 1). No active bleeding was visible. Six hours later, there was again an abrupt onset of torrential, bright red hematemesis. The bleeding did not stop before the insertion of a Sengstaken-Blakemore tube into the upper esophagus. Angiography of the thoracic aorta revealed a ruptured aneurysm in an aberrant right subclavian artery (ARSA). Because of the expected high mortality of a surgical intervention in this situation, all treatment efforts were withdrawn, and the patient died $14 \mathrm{~h}$ after admission. The autopsy showed a ruptured aneurysm of an ARSA with an arterioesophageal fistula (Figure 2).

Rupture of an ARSA aneurysm into the esophagus is rare, with only 20 reported cases [1]. Most fistulas appear as a spontaneous rupture of a preexisting atherosclerotic aneurysm in the ARSA, but there have been several reports of arterioesophageal fistulas after prolonged nasogastric esophageal intubation in young patients with an ARSA who did not have an aneurysm [2]. Survival following a ruptured ARSA with an arterioesophageal fistula has only been documented in four patients.

Fistulas between an ARSA (or another large artery) and the esophagus should be suspected when there is massive, bright red hematemesis [2]. EGD may exclude other causes of bleeding, but the sensitivity of EGD for detecting arterioesophageal fistulas is only $38 \%$ [2]. Definitive diagnosis in stable patients is achieved by computed tomography and catheter angiography [3].

\title{
Arterioesophageal fistula secondary to rupture of an aberrant right subclavian artery aneurysm: a rare differential diagnosis in upper gastrointestinal bleeding
}

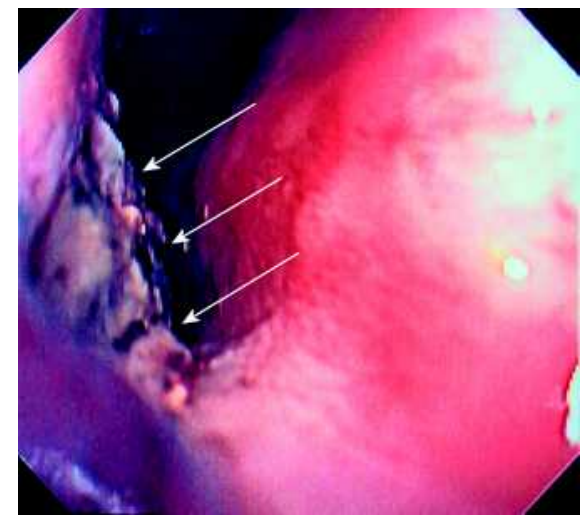

Figure 1 Endoscopic view of the esophagus. The arrows show the polypoid necrotic lesion on the dorsal wall of the esophageal mucosa.

When a suspected ARSA is eroding the esophagus and causing hemodynamic instability, vigorous resuscitation and intraesophageal balloon tamponade should be carried out immediately, followed by arteriography with optional endovascular occlusion. Surgical exploration and EGD should be conducted in the operating room [2].

Endoscopy_UCTN_Code_CCL_1AB_2AC_3AG

B. Lehmann', I. Clemetson ${ }^{2}$, A. C. Fantin ${ }^{3}$, P. Henning ${ }^{1}$, B. Kipfer ${ }^{4}$, R. Mühlethaler ${ }^{5}$,

G. Vetsch ${ }^{6}$, H.-P. Dinkel ${ }^{2}$

${ }^{1}$ Emergency Department

2 Dept. of Diagnostic Radiology

${ }^{3}$ Dept. of Gastroenterology

${ }^{4}$ Dept. of Cardiovascular Surgery

${ }^{5}$ Intensive Care Unit

${ }^{6}$ Division of Pathology, University

Hospital, Berne, Switzerland.

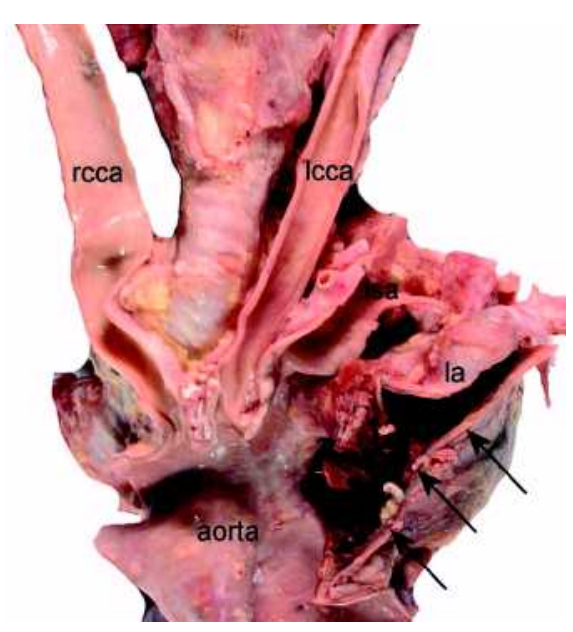

Figure 2 View of the large thoracic vessels at autopsy. The arrows show the aneurysm in the aberrant right subclavian artery. RCCA: right common carotid artery; LA: arteria lusoria; LCCA: left common carotid artery; LSA: left subclavian artery.

\section{References}

${ }^{1}$ Reynes JB, Errast CA, Ercoreca FJ, Landa AO. Aneurysm of aberrant right subclavian artery with esophageal perforation. Chest 1976; 70: 105

2 Miller RG, Robie DK, Davis SL et al. Survival after aberrant right subclavian arteryesophageal fistula: case report and literature review. J Vasc Surg 1996; 24: 271 - 275

${ }^{3}$ Kieffer E, Bahnini A, Koskas F. Aberrant subclavian artery: surgical treatment in thirtythree adult patients. J Vasc Surg 1994; 19: $100-111$

\section{Corresponding author}

\section{B. Lehmann, M.D.}

Service des Urgences

Hôpital Cantonal

1708 Fribourg

Switzerland

Fax: $\quad$ +41-26-4267316

E-mail: lehmannb@hopcantfr.ch 